\title{
The Coalsack near and far ${ }^{\star}$
}

\author{
H. Beuther ${ }^{1}$, J. Kainulainen ${ }^{1}$, Th. Henning ${ }^{1}$, R. Plume ${ }^{2}$, and F. Heitsch ${ }^{3}$ \\ 1 Max-Planck-Institute for Astronomy, Königstuhl 17, 69117 Heidelberg, Germany \\ e-mail: beuther@mpia.de \\ 2 Department of Physics and Astronomy, University of Calgary, Calgary, Canada \\ 3 Department of Physics \& Astronomy, University of North Carolina at Chapel Hill, CB 3255, Chapel Hill, NC 27599-3255, USA
}

Received 18 February 2011 / Accepted 1 July 2011

ABSTRACT

\begin{abstract}
Context. The large Coalsack dark cloud is one of the most prominent southern starless clouds, which is even visible to the naked eye. Furthermore, it is one of the rare molecular clouds without clear signs of star formation.

Aims. We investigate the dynamical properties of the gas within the Coalsack.

Methods. The two highest extinction regions were mapped with the APEX telescope in ${ }^{13} \mathrm{CO}(2-1)$ comprising a region of $\sim 1$ square degree.

Results. In addition to the well-known, nearby gas component around $-4 \mathrm{~km} \mathrm{~s}^{-1}$, we identified additional molecular gas components in particular a second extended molecular cloud at a velocity of $\sim-30 \mathrm{~km} \mathrm{~s}^{-1}$ and an estimated distance of $\sim 3.1 \mathrm{kpc}-$ that dominate the column density and visual extinction distributions in the northeastern part of the Coalsack. Although comprising $\sim 2600 M_{\odot}$, the mass of this distant cloud is distributed over an extent of $\sim 73 \mathrm{pc}$, much larger than typical high-mass infrared dark clouds. Its filamentary structure is consistent with a compressible gaseous self-gravitating cylinder, and its low mass per length indicates that it may be stable against gravitational collapse. We find barely any mid-infrared emission in archival MSX data, which is indicative of almost no star-formation activity in the near and far cloud complexes. The nearby clouds have narrow, almost thermal velocity dispersions with median values between 0.2 and $0.4 \mathrm{~km} \mathrm{~s}^{-1}$, which is also consistent with low star-formation activity. Only Tapia's Globule 2 exhibits a velocity dispersion increase toward the extinction peak and peak-velocity gradients over the core, which is indicative of a state of elevated dynamical properties.

Conclusions. The Coalsack is not one single coherent structure, but consists of several cloud complexes nearby as well as at several kpc distance. All studied clouds appear as starless low-turbulence regions that may not even collapse in the future. Only one globule exhibits more dynamical signatures and is a good candidate for present/future star formation.
\end{abstract}

Key words. stars: formation - ISM: individual objects: Coalsack - ISM: clouds - ISM: kinematics and dynamics - ISM: structure

\section{Introduction}

The Coalsack is one of the closest and most widely-recognized dark clouds in the southern hemisphere (between Galactic longitudes $305 \geq l \geq 300 \mathrm{deg}$ ) because it is visible to the bare eye (e.g., Nyman 2008). Kainulainen et al. (2009) recently produced extinction maps based on 2MASS near-infrared data of all supposedly nearby molecular clouds including the Coalsack. Figure 1 shows the large-scale extinction $\left(A_{\mathrm{v}}\right)$ map of this region. While the visual extinction through the Coalsack is generally low, there are a few regions where the extinction $A_{\mathrm{v}}$ rises above 6 and even $10 \mathrm{mag}$. Although the extinction maps set significant constraints on the morphology and column density distributions, allowing us to derive results about cloud structure and stability as well as potential column density star-formation thresholds (Kainulainen et al. 2009, 2011), these data lack any kinematical information about the cloud. Furthermore, this cloud is one of the rare cases of a nearby molecular cloud devoid of any star-formation signatures (e.g., Nyman 2008; Kainulainen et al. 2009). This is one of the reasons why it has not received as much attention in the past as typical star-forming clouds like Taurus of $\rho$ Ophiuchus. Despite this, the Coalsack constitutes an excellent laboratory to study pristine and undisturbed molecular clouds.

* The ${ }^{13} \mathrm{CO}(2-1)$ and $\mathrm{C}^{18} \mathrm{O}(2-1)$ data are available at the CDS via anonymous ftp to cdsarc.u-strasbg.fr (130.79.128.5) or via http://cdsarc.u-strasbg.fr/viz-bin/qcat?]/A+A/533/A17

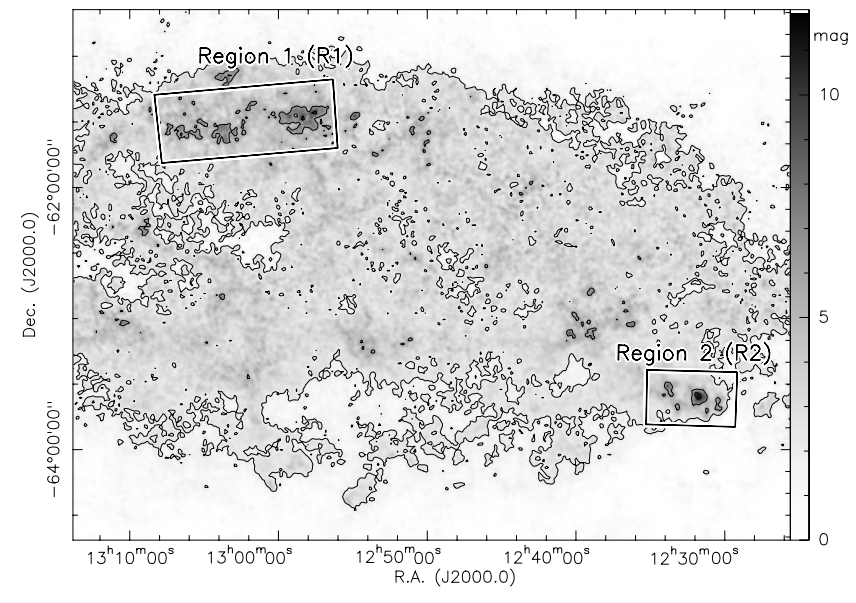

Fig. 1. 2MASS extinction map of the whole Coalsack by Kainulainen et al. (2009). The contour levels are at 2, 6 and 10 mag extinction. The two boxes outline the two regions mapped in ${ }^{13} \mathrm{CO}(2-1)$ and $\mathrm{C}^{18} \mathrm{O}(2-1)$ for this work.

Earlier spectral-line mapping on the Coalsack was conducted in ${ }^{12} \mathrm{CO}$ by Nyman et al. (1989) at a spatial resolution of $8.8^{\prime}$, and in ${ }^{13} \mathrm{CO}$ by Kato et al. (1999) at a resolution of $2.7^{\prime}$. Thus, even basic dynamical measures (e.g., central velocities, 


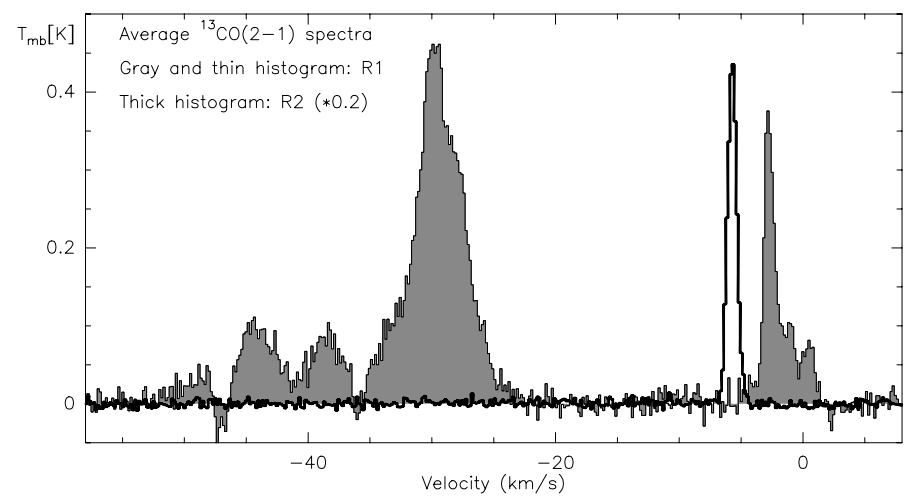

Fig. 2. Average ${ }^{13} \mathrm{CO}(2-1)$ spectra toward the northeastern region $\mathrm{R} 1$ (gray with this histogram) and the southwestern region R2 (thick histogram multiplied by 0.2 to be on the same scale).

dispersions, etc.) were limited to spatial scales larger than what is expected for molecular clumps or cores (or equally limited in dynamical range/velocity resolution). The typical velocities of the region are between -6 and $0 \mathrm{~km} \mathrm{~s}^{-1}$ (e.g., Nyman et al. 1989), and the distance estimates vary between 100 and $200 \mathrm{pc}$ (e.g., Franco 1995; Corradi et al. 1997; Knude \& Hog 1998; Nyman 2008). Nyman et al. (1989) reported the presence of an additional velocity component at $-35 \mathrm{~km} \mathrm{~s}^{-1}$, which they attribute to a background cloud associated with the Sagittarius-Carina arm that is unlikely to be responsible for any of the extinction features. Some individual fields of the Coalsack have been mapped in tracers of higher density by some authors, mainly concentrating on the region known as "Tapia's Globule 2", which presumably is one of the few dense cores in the region (e.g., Tapia 1973; Lada et al. 2004; Rathborne et al. 2009). Furthermore, Fontani et al. (2005), Beltrán et al. (2006) and Miettinen \& Harju (2010) studied a smaller sub-filament just adjacent to the eastern edge of our northeastern field. These studies, however, cover only a minuscule portion of the complex and therefore are not very informative for the dynamics of the complex itself.

To fill this missing link was one of the motivations for mapping parts of the Coalsack in spectral line emission. For this purpose, we selected the two major regions with significant fractions of cloud structure above $\sim 6$ mag extinction, marked by the two boxes in Fig. 1. The southwestern region also comprises "Tapia's Globule 2". These two regions cover in total about 1 square degree and were mapped with APEX in ${ }^{13} \mathrm{CO}(2-1)$ and $\mathrm{C}^{18} \mathrm{O}(2-1)$ at a spatial resolution of $\sim 27.5^{\prime \prime}$.

\section{Observations and data}

The $\mathrm{C}^{18} \mathrm{O}(2-1)$ and ${ }^{13} \mathrm{CO}(2-1)$ data at $219.560 \mathrm{GHz}$ and $220.399 \mathrm{GHz}$ were observed simultaneously with the Atacama Pathfinder Experiment (APEX ${ }^{1}$, Güsten et al. 2006) between April and June 2010 in the $1 \mathrm{~mm}$ band for the two regions R1 and R2 marked in Fig. 1 in on-the-fly mode. The APEX1 receiver of the SHeFI receiver family has receiver temperatures of $\sim 130 \mathrm{~K}$ at $220 \mathrm{GHz}$ (Vassilev et al. 2008), and the average system temperatures during the observations were $\sim 220 \mathrm{~K}$. Two Fast-Fourier-Transform-Spectrometer (FFTS, Klein et al. 2006) were connected, covering $\sim 2 \mathrm{GHz}$ bandwidth between 219 and $221 \mathrm{GHz}$ with a spectral resolution of $\sim 0.17 \mathrm{~km} \mathrm{~s}^{-1}$. The data

\footnotetext{
1 This publication is based on data acquired with the Atacama Pathfinder Experiment (APEX). APEX is a collaboration between the Max-Planck-Institut fur Radioastronomie, the European Southern Observatory, and the Onsala Space Observatory.
}

were converted to main-beam brightness temperatures $T_{\mathrm{mb}}$ with forward and beam efficiencies at $220 \mathrm{GHz}$ of 0.97 and 0.82 , respectively (Vassilev et al. 2008). The average $1 \sigma \mathrm{rms}$ in the maps of emission-free channels is $\sim 0.75 \mathrm{~K}$. Moment maps were conducted clipping all pixels below a $2 \mathrm{~K}$ threshold. The selected OFF-positions were at the edge of the Coalsack and were free of apparent CO emission (RA (J2000) 12:57:20.514 Dec (J2000) -60:59:43.42 for R1 and RA (J2000) 12:31:51.0 Dec (J2000) $-63: 57: 56.9$ for R2). The $F W H M$ of APEX at the given frequencies is $\sim 27.5^{\prime \prime}$.

The near-infrared extinction map is taken from Kainulainen et al. (2009). The spatial resolution of this map is $\sim 90^{\prime \prime}$.

\section{Results}

\subsection{The Coalsack: near and far components}

The ${ }^{13} \mathrm{CO}(2-1)$ emission in the northeastern region $\mathrm{R} 1$ exhibits several velocity components (see average spectrum in Fig. 2), most prominently the well-known Coalsack component at $\sim-2 \mathrm{~km} \mathrm{~s}^{-1}$ as well as a second even stronger component at $\sim-30 \mathrm{~km} \mathrm{~s}^{-1}$. Furthermore, there are additional components at more negative velocities, one centered at $\sim-38 \mathrm{~km} \mathrm{~s}^{-1}$ and one at $\sim-44 \mathrm{~km} \mathrm{~s}^{-1}$. The south-eastern region $\mathrm{R} 2$ exhibits only one velocity component at $\sim-4 \mathrm{~km} \mathrm{~s}^{-1}$. The little velocity offsets between the $\sim-2 \mathrm{~km} \mathrm{~s}^{-1}$ and $\sim-4 \mathrm{~km} \mathrm{~s}^{-1}$ components of regions R1 and R2 imply a small large-scale velocity gradient over the entire Coalsack complex. How do these components now correspond to the extinction in the Coalsack?

Figures 3 and 4 present integrated ${ }^{13} \mathrm{CO}(2-1)$ maps of the strongest emission features toward the northeastern region $\mathrm{R} 1$ and the southwestern region $\mathrm{R} 2$, respectively. While the $\sim-4 \mathrm{~km} \mathrm{~s}^{-1}$ component in region $\mathrm{R} 2$ exhibits the expected structure, peaking in the direction of the extinction peak of Tapia's globule 2 (see also Lada et al. 2004; Rathborne et al. 2009), the region R1 clearly exhibits two spatially very distinct velocity components around $\sim-2$ and $\sim-30 \mathrm{~km} \mathrm{~s}^{-1}$. Furthermore, Fig. 5 presents the integrated ${ }^{13} \mathrm{CO}(2-1)$ maps of the additional weaker components at $\sim-38 \mathrm{~km} \mathrm{~s}^{-1}$ and $\sim-44 \mathrm{~km} \mathrm{~s}^{-1}$. While we can identify spatial structures from these two velocity components as well, they are less prominent and also less extended. Therefore, we omit the latter two velocity components in the following discussion.

The $\mathrm{C}^{18} \mathrm{O}(2-1)$ emission is comparably weak and we only barely detect some structures. While we detect $\mathrm{C}^{18} \mathrm{O}(2-1)$ in the $\sim-4 \mathrm{~km} \mathrm{~s}^{-1}$ component toward R2, we only detect the $\sim-30 \mathrm{~km} \mathrm{~s}^{-1}$ component toward R1. Therefore, here we only work with the ${ }^{13} \mathrm{CO}(2-1)$ data.

Although Nyman et al. (1989) reported spectra with a second velocity component, their mentioned positions were far offset from the northeastern region R1. In addition to this, Fig. 3 clearly shows that the extinction is not caused by the nearby component at the velocity of $\sim-2 \mathrm{~km} \mathrm{~s}^{-1}$, but is mainly associated with the second much stronger velocity component at $\sim-30 \mathrm{~km} \mathrm{~s}^{-1}$. This is important because it implies that the visual dark cloud Coalsack is not one coherent low-mass cloud as believed so far, but that it is comprised of several clouds at different distances, all combined to produce the dark features on the sky. The dark features at least in the northeast come primarily from the distant component.

Following the rotation curves by either Brand \& Blitz (1993) or Reid et al. (2009), for the $-30 \mathrm{~km} \mathrm{~s}^{-1}$ component the kinematic near distances are 2.7 or $3.1 \mathrm{kpc}$, respectively. Because we see the Coalsack in extinction, we can safely take the near 


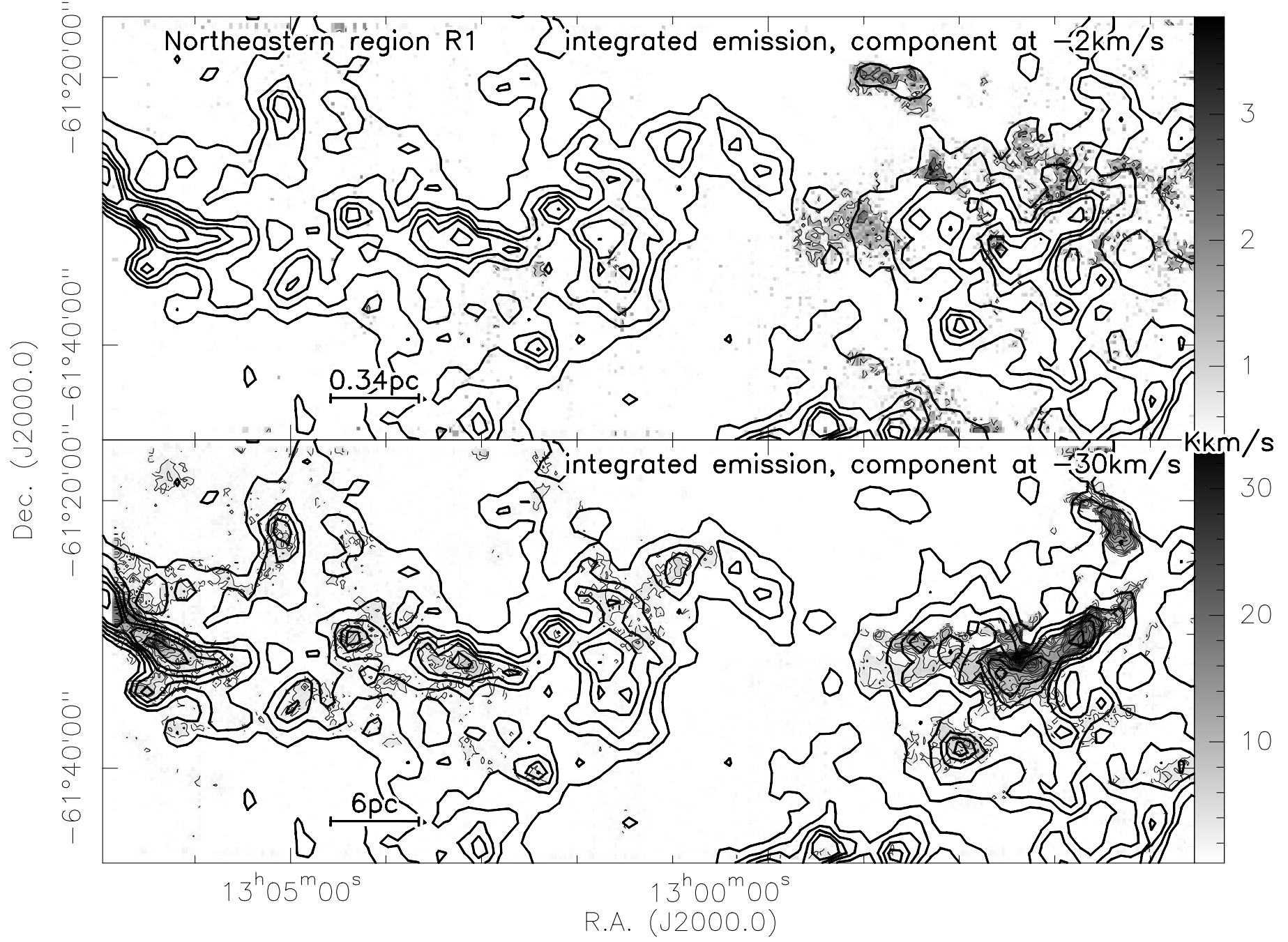

Fig. 3. Compilation of the integrated emission in grayscale (with thin contours) from the $-2 \mathrm{~km} \mathrm{~s}^{-1}$ and $-30 \mathrm{~km} \mathrm{~s}^{-1}$ components in ${ }^{13} \mathrm{CO}(2-1)$ toward the northeastern region R1 in the top and bottom panels, respectively. The thick contours outline the extinction from Kainulainen et al. (2009) in 1 mag steps between $A_{\mathrm{v}}$ of 5 and 9 mag plus additional contours of 11 and 13 mag. The integration regimes for the top and bottom panels are $[-4,2]$ and $[-35,-24] \mathrm{km} \mathrm{s}^{-1}$, respectively. The thin contours for the $-4 \mathrm{~km} \mathrm{~s}^{-1}$ map start at $1 \mathrm{~K} \mathrm{~km} \mathrm{~s}^{-1}$ and continue in $1 \mathrm{~K} \mathrm{~km} \mathrm{~s}{ }^{-1}$ steps, whereas for the $-30 \mathrm{~km} \mathrm{~s}^{-1}$ component the contours start at $2 \mathrm{~K} \mathrm{~km} \mathrm{~s}^{-1}$ and continue in $2 \mathrm{~K} \mathrm{~km} \mathrm{~s}^{-1}$ steps. A linear scale-bar is shown in both panels.

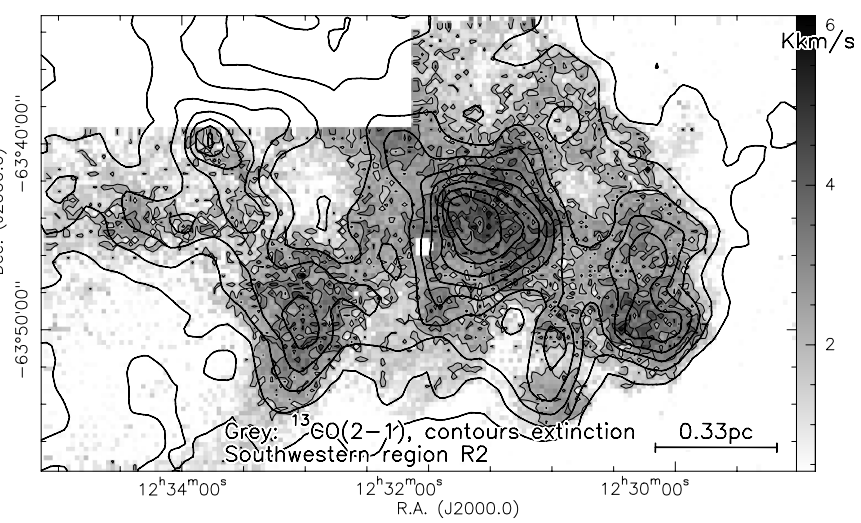

Fig. 4. The grayscale with thin contours shows the integrated ${ }^{13} \mathrm{CO}(2-1)$ emission toward the southwestern region R2. Contour levels start at $2 \mathrm{~K} \mathrm{~km} \mathrm{~s}^{-1}$ and continue in $1 \mathrm{~K} \mathrm{~km} \mathrm{~s}^{-1}$ steps. The thick contours outline the extinction from Kainulainen et al. (2009) in 1 mag steps starting at an $A_{\mathrm{v}}$ of 2 mag. A linear scale-bar is shown in the bottom-right corner.

distance since at far distances one would not see any extinction. For comparison, Fontani et al. (2005) derived a distance of

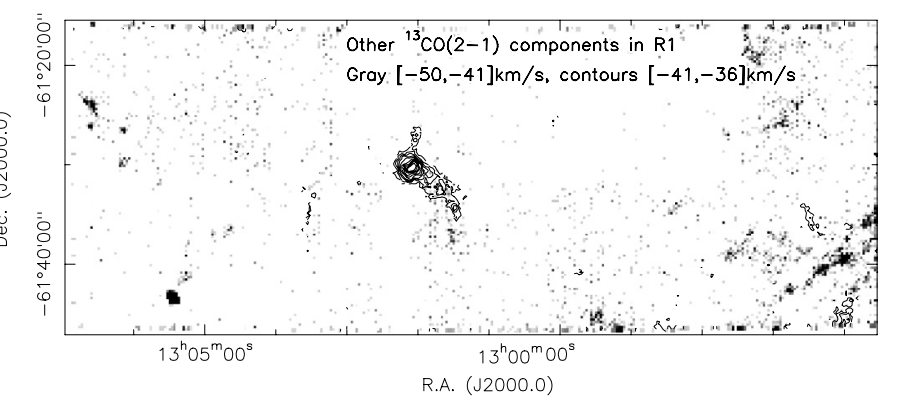

Fig. 5. Integrated ${ }^{13} \mathrm{CO}(2-1)$ images in the northern $\mathrm{R} 1$ region of the two other velocity components seen in Fig. 2. The grayscale shows the integration regime $[-41,-36] \mathrm{km} \mathrm{s}^{-1}$, and the contours present the velocity regime $[-50,-41] \mathrm{km} \mathrm{s}^{-1}$.

$2.44 \mathrm{kpc}$ for the position of the IRAS 13039-6108 source from CS data. They used a velocity of $-26.2 \mathrm{~km} \mathrm{~s}^{-1}$, consistent with our data at the eastern edge of the map (see Fig. 7), as well as the Brand \& Blitz (1993) rotation curve. Because we use an average velocity over the whole structure for the distance determination (Fig. 7) and try out different rotation curves, we will stick to the 


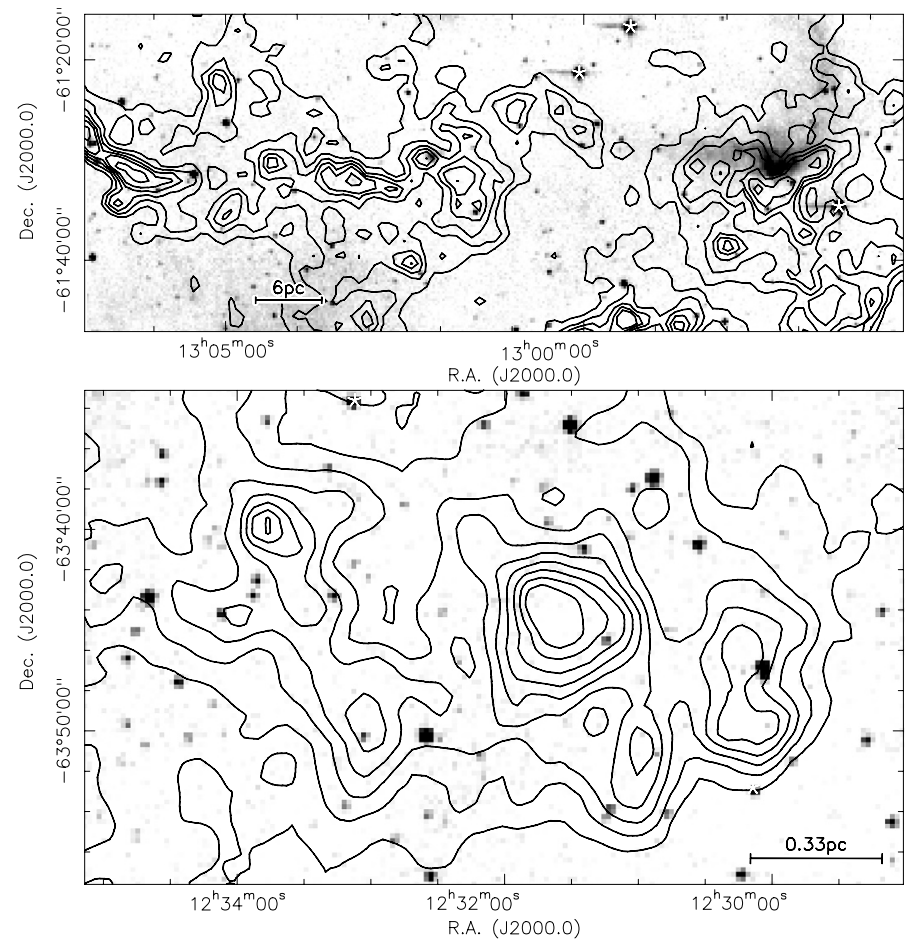

Fig. 6. The grayscaling presents the $8 \mu \mathrm{m}$ data toward the R1 (top) and R2 (bottom) regions as observed with MSX. The contours outline the extinction from Kainulainen et al. (2009) for R1 in 1 mag steps between $A_{\mathrm{v}}$ of 5 and $9 \mathrm{mag}$ plus additional contours at 11 and $13 \mathrm{mag}$, and for $\mathrm{R} 2$ in 1 mag steps starting at an $A_{\mathrm{v}}$ of 2 mag. The white stars mark the only sources that were also detected with MSX at $21 \mu \mathrm{m}$.

distance of $3.1 \mathrm{kpc}$ derived with the most recent rotation curve (Reid et al. 2009). Qualitatively speaking, these results imply that not only a small sub-filament around the IRAS source, but the whole northeastern high extinction region R1 is not, as usually assumed, a low-mass dark cloud at distances below $200 \mathrm{pc}$, but is a more massive molecular cloud at a distance of several kpc.

\subsubsection{The more massive and more distant cloud R1far}

Employing the Galactic structure outlined in Reid et al. (2009), our lines-of-sight pass through the near and far sides of the Galactic spiral arms Carina-Sagittarius and Crux-Scutum ( $\leq 2 \mathrm{kpc}$ and $\geq 3 \mathrm{kpc}$ distance, respectively). While the CarinaSagittarius arm should accordingly be responsible for the background structures of the nearby extinction cloud components, likely the Crux-Scutum arm - in which the distant cloud component should be embedded - also provides the emission against which to produce the observed extinction features.

Assuming optically thin ${ }^{13} \mathrm{CO}(2-1)$ line emission, we can calculate the gas masses for the different components following Rohlfs \& Wilson (2006). The $-30 \mathrm{~km} \mathrm{~s}^{-1}$ component in region R1 is assumed to be at a distance of $3.1 \mathrm{kpc}$ (labeled R1far from now on), whereas for the $-2 /-4 \mathrm{~km} \mathrm{~s}^{-1}$ components in R1 and R2 we use a distance of $175 \mathrm{pc}$ (and label it R1near in the northeastern region R1). Because all clouds are extinction features and hence are largely comprised of cold, mainly starless gas, an average temperature of $15 \mathrm{~K}$ appears reasonable. With these numbers, we derive a mass of $\sim 2600 M_{\odot}$ for the $-30 \mathrm{~km} \mathrm{~s}^{-1}$ $\mathrm{R} 1$ cloud, whereas the two nearby $-2 /-4 \mathrm{~km} \mathrm{~s}^{-1}$ of R1 and R2 only have masses of $\sim 1.2$ and $\sim 10.4 M_{\odot}$, respectively. Hence the
$30 \mathrm{~km} \mathrm{~s}^{-1}$ cloud at a distance of $3.1 \mathrm{kpc}$ is $2-3$ orders of magnitude more massive than the other two components at the more typical Coalsack distance. However, one should keep in mind that a $\sim 2600 M_{\odot}$ cloud, that is distributed over a size of $\sim 73 \mathrm{pc}$ in east-west direction, cannot be considered as a typical high-mass star-forming region. The mass is quite smoothly distributed over a large spatial area. For comparison, cloud masses of typical lowand high-mass star-forming regions like Taurus or Orion vary between several thousand to several $10^{4} M_{\odot}$ (e.g., Kainulainen et al. 2009).

To check for signatures of ongoing star formation, we investigated the 8 and $21 \mu \mathrm{m}$ images of the MSX satellite (Price $1995)^{2}$. Figure 6 presents overlays of the $8 \mu \mathrm{m}$ emission with the extinction map. The northern region $\mathrm{R} 1$ shows one bright $8 \mu \mathrm{m}$ source with a bit of extended diffuse emission in the west. This bright region may be associated with the extinction peak at an approximate separation of 2.6", however, no $21 \mu \mathrm{m}$ emission is detected from that region. While the $8 \mu \mathrm{m}$ peak is neither clearly associated with the $-30 \mathrm{~km} \mathrm{~s}^{-1}$ nor with the $-2 \mathrm{~km} \mathrm{~s}^{-1}{ }^{13} \mathrm{CO}$ component, the more diffuse $8 \mu \mathrm{m}$ emission extending from this peak toward the north-west is associated with near-infrared extinction as well as ${ }^{13} \mathrm{CO}(2-1)$ emission of the $-30 \mathrm{~km} \mathrm{~s}^{-1}$ component. Therefore, it appears more likely that the $8 \mu \mathrm{m}$ peak is also associated with the $-30 \mathrm{~km} \mathrm{~s}^{-1}$ component. In general, there is barely any $21 \mu \mathrm{m}$ emission detected toward both regions, implying no or only a marginal level of embedded star formation. This is consistent with the general picture of the Coalsack being largely a starless cloud.

\subsection{Kinematic analysis of the Coalsack clouds}

In addition to the distance and mass estimates, the ${ }^{13} \mathrm{CO}(2-1)$ data also allow us to characterize the kinematic properties of the two - or more correctly three - regions. Here, we will focus on the northeastern regions (R1far and R1near) and the southwestern region $\mathrm{R} 2$ separately.

Figure 7 presents the ${ }^{13} \mathrm{CO}(2-1)$ first-moment maps (intensity-weighted peak velocities) of the two northeastern regions R1far and R1near. Covering an east-west length of $\sim 81^{\prime}$ (or $\sim 1.35 \mathrm{deg}$ ) equivalent to a linear extent $\sim 73 \mathrm{pc}$, the velocity gradient across this structure is relatively small with a velocity difference of only $\sim 8 \mathrm{~km} \mathrm{~s}^{-1}$ (from $\sim-26$ to $\sim-34 \mathrm{~km} \mathrm{~s}^{-1}$ ), corresponding to $\sim 0.1 \mathrm{~km} \mathrm{~s}^{-1} \mathrm{pc}^{-1}$. The relatively smooth velocity gradient implies that this is one coherent structure, even the parts with lower extinction and almost no ${ }^{13} \mathrm{CO}(2-1)$ detection in the middle of the filamentary structure.

Inspired by the recent work of Jackson et al. (2010) on the large ( $\sim 80$ pc length) "Nessie" filamentary infrared dark cloud (IRDC), we investigated whether the $-30 \mathrm{~km} \mathrm{~s}^{-1}$ component R1far may also be consistent with a gravitationally bound gaseous cylinder that may fragment because of fluid instabilities. For that purpose we used the clumpfind algorithm (Williams et al. 1994) to extract clump peak positions from the nearinfrared extinction map. Because we are only interested in the peak positions here, we started with relatively high 6 mag contours and continued at $1 \mathrm{mag}$ levels as input parameters for clumpfind. Because the filamentary structure is a bit larger than our ${ }^{13} \mathrm{CO}$ coverage, we used also a slightly larger extinction map for the purpose of the filament analysis (Fig. 8). The projected east-west extent of the analyzed structure is $\sim 88 \mathrm{pc}$. The

\footnotetext{
${ }^{2}$ Our Coalsack targets are only barely covered by Spitzer because they are at relatively high and low galactic longitude, and GLIMPSE only covered latitudes $\leq \pm 1$ deg (Churchwell et al. 2009).
} 


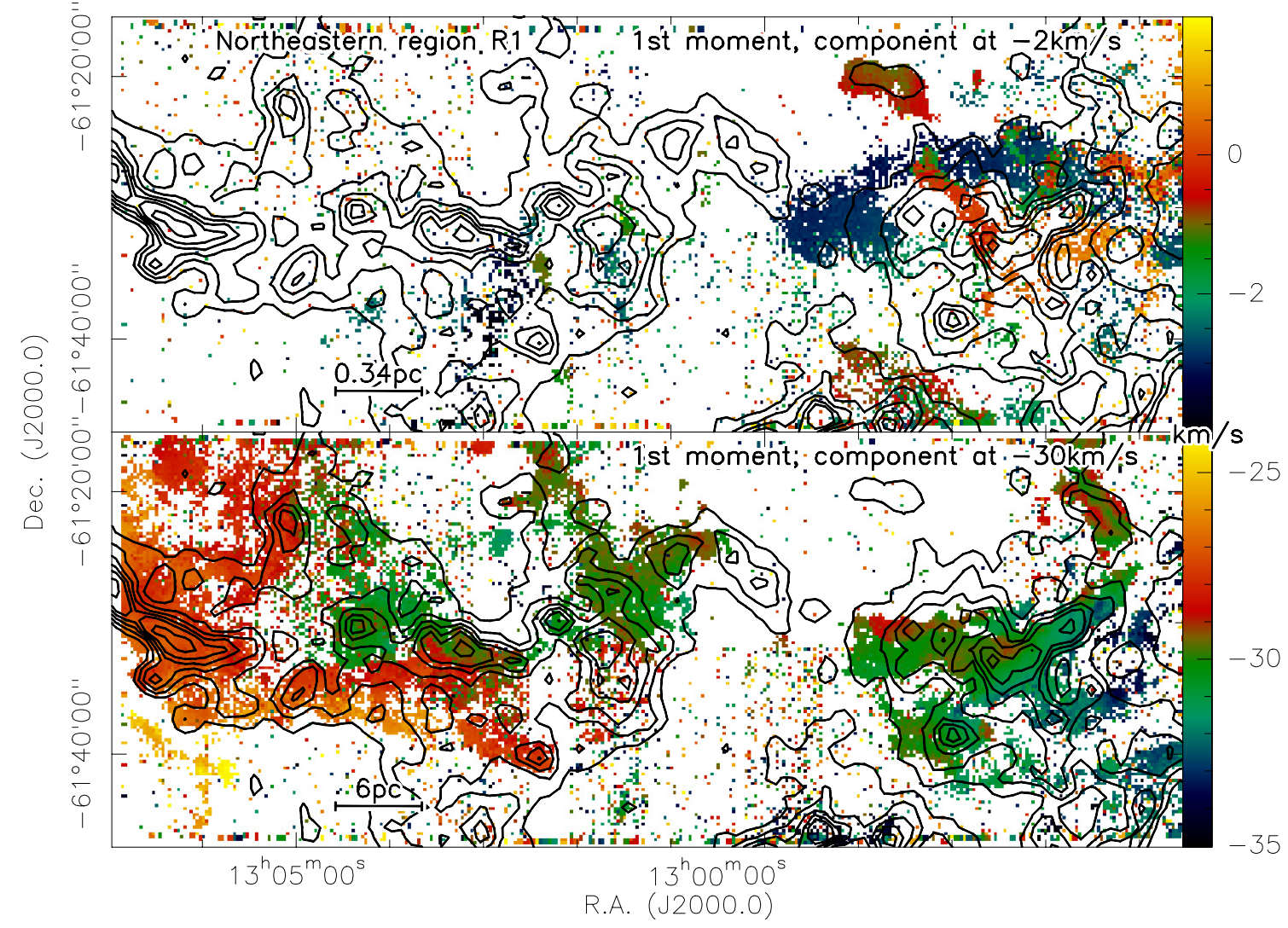

Fig. 7. Compilation of ${ }^{13} \mathrm{CO}(2-1)$ 1st moment maps toward the northeastern region R1 as marked in the panels. The top and bottom panels show the -2 and $-30 \mathrm{~km} \mathrm{~s}^{-1}$, respectively. The thick contours outline the extinction from Kainulainen et al. (2009) in 1 mag steps starting between $A_{\mathrm{v}}$ of 5 and 9 mag plus additional contours at 11 and 13 mag. Linear scale-bars are shown in the bottom-left corner of each panel.

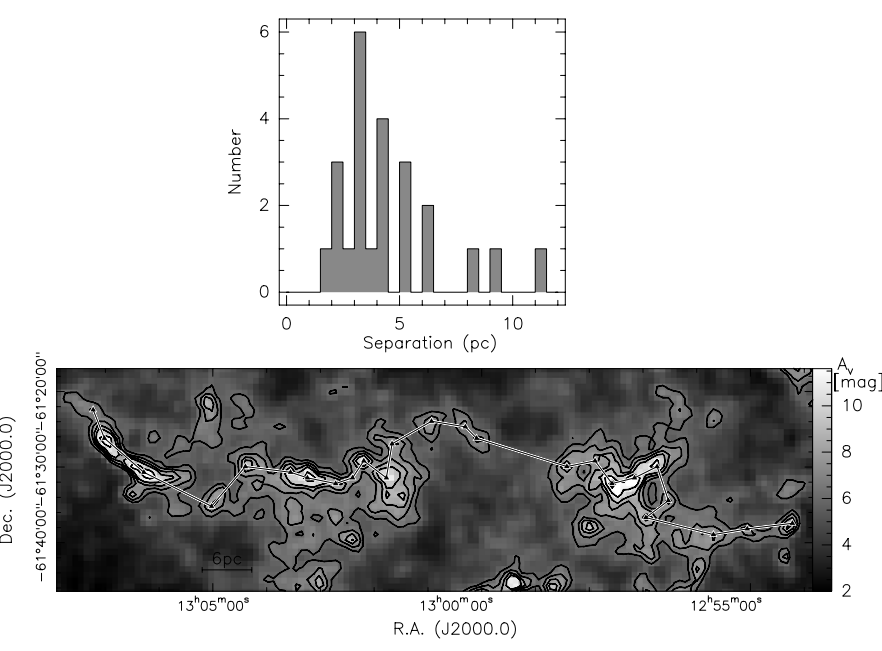

Fig. 8. Outline of the clump distribution for R1far. The grayscale with contours shows the extinction map from Kainulainen et al. (2009) in 1 mag steps between $A_{v}$ of 6 to 9 mag plus additional contours at 11 and $13 \mathrm{mag}$. A linear scale-bar is shown at the bottom-left. The east-west extent of this extinction map is slightly larger than that of the spectral line data. The triangles mark the clump peak positions as derived via gaussclump (Williams et al. 1994), and the connecting line visualizes the separations measured between the clumps. The histogram above shows the distribution of nearest neighbor clump separations as derived from the extinction map.

extracted positions are shown in Fig. 8. Based on these peak positions we calculated the projected linear separations for these peaks assuming a distance of $3.1 \mathrm{kpc}$. One should keep in mind that all observed separations discussed below have always to be considered as lower limits because we have no information about the inclination angle of the filament with the plane of the sky. Figure 8 also presents a histogram of the distribution of linear separations, and the peak of the distribution is at about $\sim 3.5 \mathrm{pc}$. While the mean separation is $\sim 4.6 \mathrm{pc}$, the median is at $\sim 4.1 \mathrm{pc}$. This separation is resolved well by the spatial resolution of the extinction map of $\sim 1.35 \mathrm{pc}$, and random placement of the cores within the filament would produce a very different distribution with a mean core separation of $\sim 2$ pc. The median separation within the R1far filament is consistent with that derived for the "Nessie" IRDC by Jackson et al. (2010). For more discussion see Sect. 4.1.

Figure 9 presents the 2nd moment maps - or the intensityweighted velocity dispersion. While there is a spread in velocity dispersion between approximately 0.4 and $4 \mathrm{~km} \mathrm{~s}^{-1}$ over the entire region, most observed line-widths are at the lower end of the distribution with an average velocity dispersion over the entire field of $\sim 1.26 \mathrm{~km} \mathrm{~s}^{-1}$ and a median velocity dispersion of $\sim 0.8 \mathrm{~km} \mathrm{~s}^{-1}$. This relatively narrow average line-width compared to more evolved star-forming regions confirms the previous assessment from the near- and mid-infrared data, that the whole region exhibits low star-formation activity capable of contributing to the line-width broadening.

\subsubsection{The low-mass nearby cloud R1near}

Similar to R1far, Figs. 7 and 9 also present the 1st and 2nd moment maps of the $-2 \mathrm{~km} \mathrm{~s}^{-1}$ component in the $\mathrm{R} 1$ region. From now on we call that R1near. As already outlined in Sect. 3.1, 


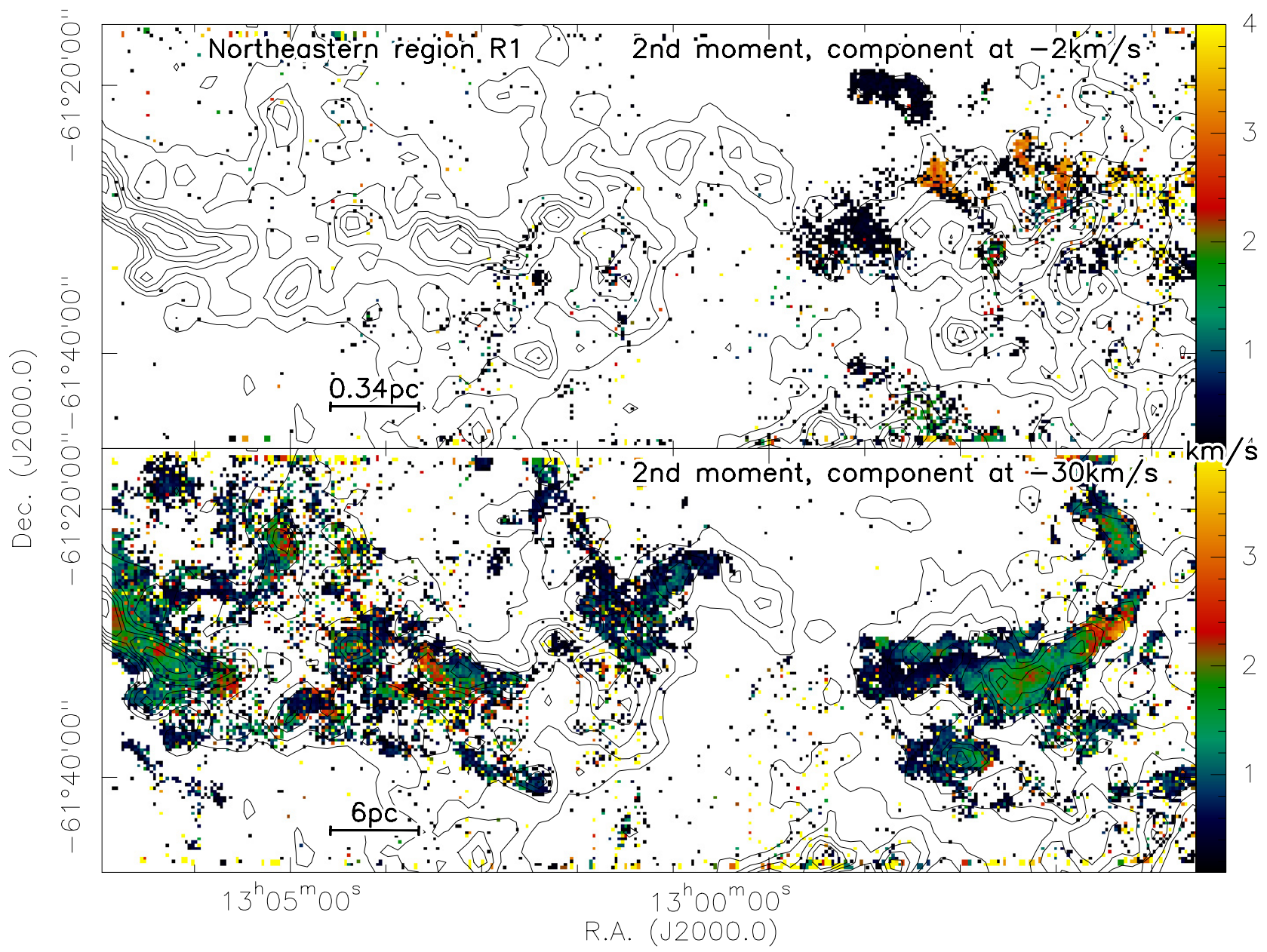

Fig. 9. Compilation of ${ }^{13} \mathrm{CO}(2-1)$ 2nd moment maps toward the northeastern region $\mathrm{R} 1$ as marked in the panels. The top and bottom panels show the -2 and $-30 \mathrm{~km} \mathrm{~s}^{-1}$, respectively. The thick contours outline the extinction from Kainulainen et al. (2009) in 1 mag steps between $A_{\mathrm{v}}$ of 5 and 9 mag plus additional contours at 11 and 13 mag. Linear scale-bars are shown in the bottom-left corner of each panel.

there is barely any spatial association of the extinction map with the $-2 \mathrm{~km} \mathrm{~s}^{-1}{ }^{13} \mathrm{CO}(2-1)$ emission. The velocity spread of the region between RA 13:00:00 and the western edge of the map is only about $4 \mathrm{~km} \mathrm{~s}^{-1}$ between $\sim-3$ and $\sim 1 \mathrm{~km} \mathrm{~s}^{-1}$. This velocity gradient covers an east-west extent of $\sim 32^{\prime}$, corresponding at a distance of $175 \mathrm{pc}$ to an approximate linear extent of $\sim 1.6 \mathrm{pc}$. This results in an approximate velocity gradient of $2.6 \mathrm{~km} \mathrm{~s}^{-1} \mathrm{pc}^{-1}$. While this velocity spread appears to be relatively small compared with the linear velocity gradient one can derive for $\mathrm{R} 1 \mathrm{far}\left(\sim 0.1 \mathrm{~km} \mathrm{~s}^{-1} \mathrm{pc}^{-1}\right)$, it is still comparatively large. Or the other way round, the velocity gradient for R1far is extremely small over a much larger spatial extent (see previous section).

The 2nd moment or velocity dispersion distribution for R1near is even narrower than that for R1far (Fig. 9). While there are a few small regions exhibiting velocities $>1 \mathrm{~km} \mathrm{~s}^{-1}$, the average and median velocity dispersions for R1near are $0.85 \mathrm{~km} \mathrm{~s}^{-1}$ and $0.2 \mathrm{~km} \mathrm{~s}^{-1}$, respectively. While the average represents the spread in velocities a bit better, the median clearly shows that most observed line-widths are very narrow. At an approximate cold temperature of $15 \mathrm{~K}$, the thermal line-width of ${ }^{13} \mathrm{CO}(2-1)$ is $0.15 \mathrm{~km} \mathrm{~s}^{-1}$, which closely agree with the observed median velocity dispersion of this nearby cloud. Hence, R1near resembles a cold and mainly starless cloud with an almost thermal velocity dispersion.

\subsubsection{The Coalsack cloud R2 comprising Tapia's Globule 2}

Figure 10 presents the 1 st and 2nd moment maps (intensityweighted peak-velocities and velocity dispersion) for the southwestern region R2, also known as "Tapia's Globule 2" (Tapia 1973; Lada et al. 2004; Rathborne et al. 2009). The peak-velocity distribution in this region does not exhibit as clear a velocity gradients as seen in R1far and R1near (see previous sections), but we find velocity gradients from the east and west of the main globule toward the extinction peak. East and west of the main peak, the $1 \mathrm{st}$ moment map reveals peak velocities $\sim-5.8 \mathrm{~km} \mathrm{~s}^{-1}$, whereas in a relatively broad north-south filament extending almost across the central extinction peak, the peak velocities are found to be around $-5.5 \mathrm{~km} \mathrm{~s}^{-1}$. Hence, in these ${ }^{13} \mathrm{CO}(2-1)$ data we do not recover the east-west velocity gradient previously discussed by Rathborne et al. (2009) for the inner $6^{\prime} \times 6^{\prime}$ around the main extinction peak.

The velocity dispersion of this region is also very narrow, spanning a range mainly below $1 \mathrm{~km} \mathrm{~s}^{-1}$ (Fig. 10 bottom panel). In contrast to the $\mathrm{R} 1$ region where the average and mean values of the velocity dispersions deviated from each other, which indicatis a broader velocity spread, for $\mathrm{R} 2$, the average and mean velocity dispersion are approximately the same with $\sim 0.4 \mathrm{~km} \mathrm{~s}^{-1}$. This can be interpreted as a sign of a relatively uniform velocity dispersion over the region. While the velocity dispersion at the 


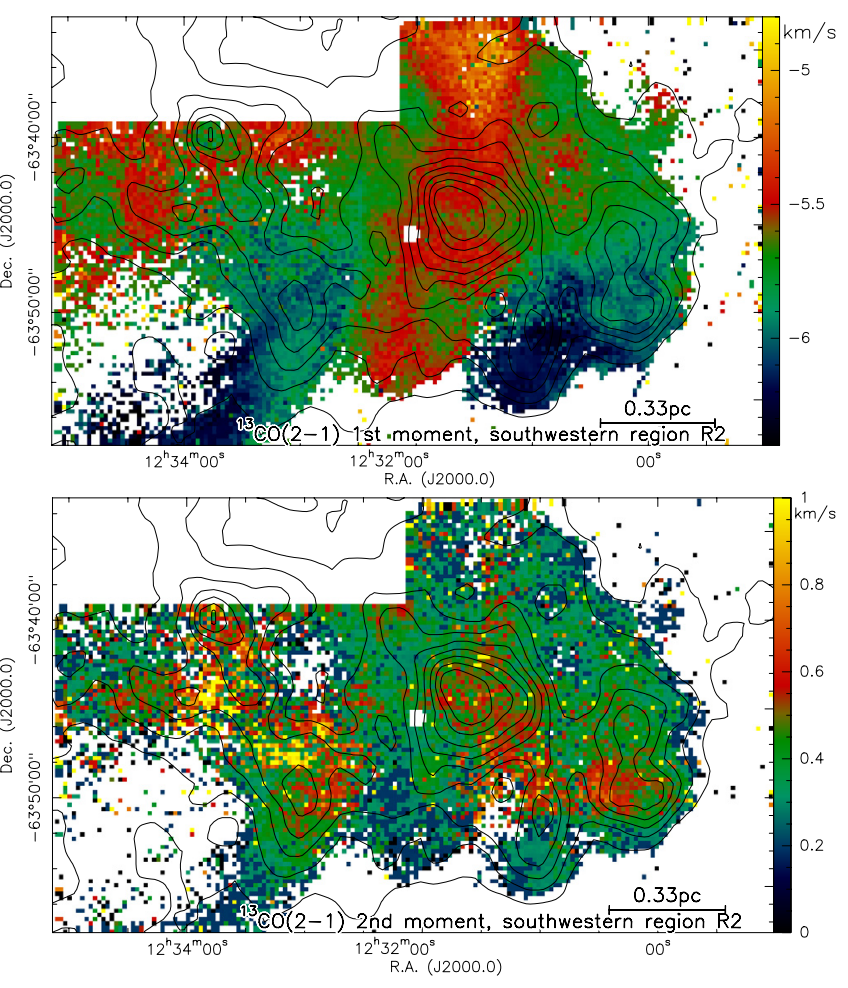

Fig. 10. Compilation of ${ }^{13} \mathrm{CO}(2-1)$ 1st and 2nd moment maps toward the southwestern region R2 in the top and bottom panel, respectively. The thick contours outline the extinction from Kainulainen et al. (2009) in 1 mag steps starting at an $A_{\mathrm{v}}$ of 2 mag. Linear scale-bars are shown in the bottom-right corner of each panel.

edge of the map is around $0.2 \mathrm{~km} \mathrm{~s}^{-1}$, in the outskirts of the main extinction peak it rises to values on the order of $\sim 0.4 \mathrm{~km} \mathrm{~s}^{-1}$, and toward the extinction peak one finds values on the order of of $0.6 \mathrm{~km} \mathrm{~s}^{-1}$ or a bit higher. While even the latter values are still narrow compared to more active star-forming regions (e.g., Goldsmith et al. 2008), they nevertheless are indicative of nonthermal gas motions in the direction of the highest extinction. For a more detailed discussion about the dynamical properties of the region, see Sect. 4.2.

\section{Discussion}

\subsection{Fragmenting filaments}

The overall extent of the two clouds - "Nessie" discussed in Jackson et al. (2010) and R1far presented here - is similar with $\sim 80 \mathrm{pc}$ and $\sim 88 \mathrm{pc}$, respectively, and they are at similar distances (both at $\sim 3.1 \mathrm{kpc}$ ). However, there are also considerable differences between the two clouds. First of all, the length-to-width ratio is different because while "Nessie" is extremely narrow with an approximate cylinder radius $r_{\text {cyl }}$ of only $\sim 0.5 \mathrm{pc}$, the structure within R1far is less well defined and hence it is also more difficult to accurately determine a radius. However, based on the extinction maps, we roughly estimate an approximate average radius $r_{\text {cyl }}$ of $\sim 2-2.5$ pc for R1far, about four to five times larger than that of "Nessie", derived from their MIPSGAL midinfrared emission. Furthermore, Jackson et al. (2010) report that star-formation activity is clearly identified toward many of their clumps, while our analysis reveals barely any star formation activity toward R1far. In addition to this, as discussed in Sect. 3.1, because the mass of R1far is distributed over such a large spatial extent, it cannot be considered as a "typical" very young highmass star-forming region or IRDC.
How does this structure compare to a gravitationally bound isothermal gaseous cylinder? Following Jackson et al. (2010) as well as the original work by Chandrasekhar \& Fermi (1953), Nagasawa (1987) and Inutsuka \& Miyama (1992), we try to estimate characteristic fragmentation scales for different conditions within self-gravitating gas cylinders. For an incompressible fluid, a characteristic length scale can be defined as $\lambda_{\text {frag }} \approx 11 r_{\text {cyl }}$, whereas for an infinite isothermal gas cylinder, the relation is $\lambda_{\text {frag }} \approx 22 H$ where $H$ is the isothermal scale height given by $H=c_{\mathrm{s}}(4 \pi G \rho)^{-1 / 2}$ with $c_{\mathrm{s}}$ the sound speed, $G$ the gravitational constant and $\rho$ the central gas mass density.

Depending on the sound speed $c_{\mathrm{s}}$ we can calculate the characteristic scale height of such a filament. With a thermal sound speed of the gas at $15 \mathrm{~K}$ of $c_{\mathrm{s}} \sim 0.23 \mathrm{~km} \mathrm{~s}^{-1}$ and using $n_{\text {crit }} \sim$ $9 \times 10^{3} \mathrm{~cm}^{-3}$ as a proxy for the density, the characteristic scaleheight is $H \approx 0.07 \mathrm{pc}$. However, inspecting Fig. 9, we find that the region rather exhibits an average velocity dispersion of $\sim 1.26 \mathrm{~km} \mathrm{~s}^{-1}$, which we use as a proxy for the full-widthhalf-maximum $F W H M$ (see Sect. 3.1.1). If one now replaces the sound speed $c_{\mathrm{s}}$ with the Gaussian velocity dispersion $\sigma=$ $1 / 2.35 * F W H M$, we obtain a higher scale-height of $H \approx 0.16 \mathrm{pc}$. This implies that the approximate cylinder radius $r_{\mathrm{cyl}} \sim 2.25 \mathrm{pc}$ in any case exceeds the characteristic scale height by more than an order of magnitude. Because the characteristic length scale $\lambda_{\text {frag }} \approx 11 r_{\text {cyl }}$ for an incompressible fluid requires $r_{\text {cyl }} \ll H$ (e.g., Jackson et al. 2010), the incompressible case can safely be excluded for R1far.

Looking at the characteristic fragmentation scale for the infinite isothermal gas cylinder $\lambda_{\text {frag }} \approx 22 \mathrm{H}$, we derive $\lambda_{\text {frag }} \approx$ $1.46 \mathrm{pc}$ or $\lambda_{\text {frag }} \approx 3.5 \mathrm{pc}$ for the sound speed $c_{\mathrm{s}}$ or the Gaussian velocity dispersion $\sigma$, respectively. Because our data are clearly not described by a pure thermal line-width (Fig. 9), the fragmentation scale $\lambda_{\text {frag }} \approx 3.5$ pc for a Gaussian velocity dispersion consistent with our data appears the most reasonable characteristic length scale we can derive in the framework of this picture of a gravitationally bound isothermal gaseous cylinder.

Comparing this latter $\lambda_{\text {frag }} \approx 3.5 \mathrm{pc}$ with our derived median clump separation of $\sim 4$ pc (Sect. 3.1.1), the data are broadly consistent with a compressible gravitationally bound isothermal gaseous cylinder, in particular if one considers the observed spread in velocity dispersion values as well as an intrinsic spread in the gas densities. However, they are inconsistent with an incompressible fluid.

Another way to characterize self-gravitating cylinders is in the framework of maximum critical mass per unit length $M / l_{\max }$ along the cylinder's axis (Ostriker 1964; Fiege \& Pudritz 2000a,b). Above the maximum critical linear mass density, the cylinders would be doomed to collapse. Following again Jackson et al. (2010) and using the mean ${ }^{13} \mathrm{CO}(2-1)$ line-width of $\Delta v \approx$ $1.26 \mathrm{~km} \mathrm{~s}^{-1}$, for turbulently supported filaments we find a maximum critical $M / l_{\max }=84(\Delta v)^{2} M_{\odot} \mathrm{pc}^{-1}=133 M_{\odot} \mathrm{pc}^{-1}$ (about a factor 10 higher than the maximum critical $M / l_{\max } \sim 15 M_{\odot} \mathrm{pc}^{-1}$ for undisturbed thermal filaments, Ostriker 1964; Inutsuka \& Miyama 1997). To compare that with our observations, we can obtain a rough estimate of the observed $M / l$ by dividing the total mass estimated from the ${ }^{13} \mathrm{CO}$ data in Sect. $3.1\left(\sim 2600 M_{\odot}\right)$ by the approximate length of the filament of $\sim 73 \mathrm{pc}$ in the ${ }^{13} \mathrm{CO}$ data. This results in an approximate observed $M / l$ of only $\sim 36 M_{\odot} \mathrm{pc}^{-1}$. Although this is larger than the maximum critical linear mass density for thermal filaments (and also the measured $M / l$ ratio in, e.g., a thermal filament in Taurus, Schmalzl et al. 2010), the observed mass-per-length scale is approximately a factor 4 below the maximum critical $M / l_{\max }$ ratio for turbulently supported filaments based on the ${ }^{13} \mathrm{CO}(2-1)$ line-width. While 
a lower than critical $M / l_{\max }$ does not necessarily imply stability against collapse, it is at least consistent with the Coalsack at large not (or only barely) forming stars at the moment.

Comparing our results with those from the literature, we find that in the framework of isothermal gravitationally bound cylinders, we can distinguish for R1far between a compressible and an incompressible configuration, whereas this is not possible for the "Nessie" IRDC (Jackson et al. 2010). Furthermore, the $M / l$ ratio for R1far with $\sim 36 M_{\odot} \mathrm{pc}^{-1}$ is considerably lower than that for "Nessie" or Orion $\left(\sim 110 M_{\odot} \mathrm{pc}^{-1}\right.$ and $\sim 385 M_{\odot} \mathrm{pc}^{-1}$, respectively, Jackson et al. 2010). While one cannot directly compare the two latter values with the threshold we estimated above because the observed line-width in these clouds are also larger than in the Coalsack, nevertheless, this comparison confirms the expected evolution that starless clouds have low $M / l$ ratios that increase with time during the collapse and star-formation processes. However, not all low $M / l$ clouds have to evolve like that. They could also be transient structures that are dispersing again.

\subsection{Dynamical properties of R2/Tapia's Globule 2}

Rathborne et al. (2009) recently suggested that the ring-like structure within this globule may have formed from the merging of two sub-sonic flows. In contrast to Rathborne et al. (2009), we do not find a velocity gradient across the globule. This discrepancy likely arises from different observational biases, e.g., we observed the region in ${ }^{13} \mathrm{CO}$, whereas Rathborne et al. (2009) employed the rarer isotopologue $\mathrm{C}^{18} \mathrm{O}$. Furthermore, their $\mathrm{C}^{18} \mathrm{O}$ map covered a much smaller area of the globule, and if one focuses on the central few arcminutes of our data (Fig. 10, top panel), one could also get the impression of a velocity gradient. Nevertheless, the velocity dispersion increase toward the center is suggestive of dynamical evolution within the core. To further investigate the dynamics of the region, we produced a centralvelocity-increment (CVI, e.g., Hily-Blant et al. 2008) map of the region, which is a different representation of the velocity changes over the observed region. Because the velocity structure is more prominent in east-west direction, the CVI map presented in Fig. 11 studies the velocity changes in the RA direction with 10 pixel step sizes, corresponding to $\sim 143^{\prime \prime}$ or approximately five resolution elements (Sect. 2). The observed peak-velocity changes are small toward the central peak of the extinction map, however, we see four enhancements of the CVI-values toward the eastern and western edges in the north and south of the main extinction peak. This is consistent with the fact that we see a filamentary structure going from north to south through Globule 2 in the 1st moment map (Fig. 10). Returning to the merging subsonic flow picture by Rathborne et al. (2009), our data do not completely confirm this picture because we do not see a clear east-west velocity gradient across as Rathborne et al. (2009) do. However, the combined facts that we find clear CVI increases at the Globule's edges as well as an increase of velocity dispersion toward the extinction peak are strongly suggestive of a dynamically active state of this dark globule within the large Coalsack complex. From these data we cannot establish the exact reason for the dynamical evolution, however, this Globule is an excellent candidate region for future active collapse and starformation processes.

\section{Conclusions}

Mapping approximately 1 square degree around the regions of the highest extinction in the almost entirely starless Coalsack

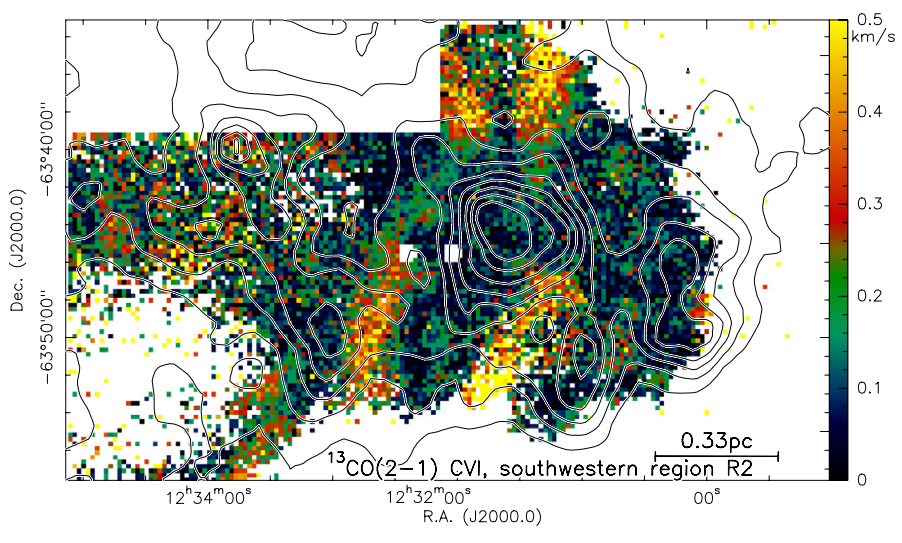

Fig. 11. ${ }^{13} \mathrm{CO}(2-1)$ central velocity increment $(\mathrm{CVI})$ in RA toward the southwestern region $\mathrm{R} 2$. The thick contours outline the extinction from Kainulainen et al. (2009) in 1 mag steps starting at an $A_{\mathrm{v}}$ of 2 mag. A linear scale-bar is shown in the bottom-right corner.

dark cloud in ${ }^{13} \mathrm{CO}(2-1)$ with APEX revealed several interesting properties:

- The Coalsack is not one coherent dark low-mass cloud at distances below $200 \mathrm{pc}$ and typical velocities of $\sim-4$ to $0 \mathrm{~km} \mathrm{~s}^{-1}$, but we find a second velocity component at $\sim-30 \mathrm{~km} \mathrm{~s}^{-1}$ corresponding to a second cloud at a distance of $\sim 3.1 \mathrm{kpc}$. This second more distant cloud dominates the dust-extinction signatures in the northeast of the Coalsack.

- Mid-infrared MSX data reveal barely any star-formation activity toward any of the mapped clouds.

- Although the total observed mass of the distant cloud with $2600 M_{\odot}$ is far larger than those of the two mapped nearby clouds (on the order of several solar masses), we cannot consider it as a potential high-mass star-forming region, because its mass is distributed over a spatial extent in east-west direction of $\sim 73 \mathrm{pc}$. Hence, it is smoothly distributed over a large spatial area.

- The more distant cloud resembles in shape a large and twisted filament. Analyzing this structure within the framework of an isothermal gravitationally bound cylinder, we find that the data are consistent with a compressible gaseous filament and inconsistent with an incompressible fluid. Furthermore, the characteristic mass per length $M / l$ is low with $\sim 36 M_{\odot} \mathrm{pc}^{-1}$. Hence, this structure is potentially stable against gravitational collapse.

- The nearby low-mass clouds both exhibit narrow velocity distributions with median values between 0.2 and $0.4 \mathrm{~km} \mathrm{~s}^{-1}$, also indicative of almost no star-formation activity.

- Only Tapia's Globule 2 appears different. While the peak velocity dispersion barely exceeds $0.6 \mathrm{~km} \mathrm{~s}^{-1}$, we nevertheless see a line-width increase from the map edge toward the extinction peak. Furthermore, an analysis of the centralvelocity-increments reveals significant velocity changes at the Globule's edges. Both features are suggestive of dynamical action in that region, e.g., maybe early infall activity.

In summary, our data reveal different cloud components nearby as well as far away. While all observed clouds are mostly bare of any star-formation activity, we find filamentary structures consistent with compressible self-gravitating gaseous cylinders as well as one Globule in an apparent elevated dynamical state.

Acknowledgements. This research made use of data products from the Midcourse Space Experiment. Processing of the data was funded by the Ballistic Missile Defense Organization with additional support from NASA Office of 
Space Science. This research has also made use of the NASA/IPAC Infrared Science Archive, which is operated by the Jet Propulsion Laboratory, California Institute of Technology, under contract with the National Aeronautics and Space Administration.

\section{References}

Beltrán, M. T., Brand, J., Cesaroni, R., et al. 2006, A\&A, 447, 221 Brand, J., \& Blitz, L. 1993, A\&A, 275, 67

Chandrasekhar, S., \& Fermi, E. 1953, ApJ, 118, 116

Churchwell, E., Babler, B. L., Meade, M. R., et al. 2009, PASP, 121, 213

Corradi, W. J. B., Franco, G. A. P., \& Knude, J. 1997, A\&A, 326, 1215

Fiege, J. D., \& Pudritz, R. E. 2000a, MNRAS, 311, 85

Fiege, J. D., \& Pudritz, R. E. 2000b, MNRAS, 311, 105

Fontani, F., Beltrán, M. T., Brand, J., et al. 2005, A\&A, 432, 921

Franco, G. A. P. 1995, A\&AS, 114, 105

Goldsmith, P. F., Heyer, M., Narayanan, G., et al. 2008, ApJ, 680, 428

Güsten, R., Nyman, L. A., Schilke, P., et al. 2006, A\&A, 454, L13

Hily-Blant, P., Falgarone, E., \& Pety, J. 2008, A\&A, 481, 367

Inutsuka, S., \& Miyama, S. M. 1992, ApJ, 388, 392

Inutsuka, S., \& Miyama, S. M. 1997, ApJ, 480, 681

Jackson, J. M., Finn, S. C., Chambers, E. T., Rathborne, J. M., \& Simon, R. 2010, ApJ, 719, L185
Kainulainen, J., Beuther, H., Henning, T., \& Plume, R. 2009, A\&A, 508, L35

Kainulainen, J., Beuther, H., Banerjee, R., Federrath, C., \& Henning, T. 2011, A\&A, 530, A64

Kato, S., Mizuno, N., Asayama, S., et al. 1999, PASJ, 51, 883

Klein, B., Philipp, S. D., Krämer, I., et al. 2006, A\&A, 454, L29

Knude, J., \& Hog, E. 1998, A\&A, 338, 897

Lada, C. J., Huard, T. L., Crews, L. J., \& Alves, J. F. 2004, ApJ, 610, 303

Miettinen, O., \& Harju, J. 2010, A\&A, 520, A102

Nagasawa, M. 1987, Prog. Theor. Phys., 77, 635

Nyman, L. A. 2008, Handbook of Star Forming Regions, 2

Nyman, L., Bronfman, L., \& Thaddeus, P. 1989, A\&A, 216, 185

Ostriker, J. 1964, ApJ, 140, 1529

Price, S. D. 1995, Space Sci. Rev., 74, 81

Rathborne, J. M., Lada, C. J., Walsh, W., Saul, M., \& Butner, H. M. 2009, ApJ, 690, 1659

Reid, M. J., Menten, K. M., Zheng, X. W., et al. 2009, ApJ, 700, 137

Rohlfs, K., \& Wilson, T. L. 2006, Tools of radio astronomy, 4th rev. and enl., ed. K. Rohlfs, \& T.L. Wilson. ( Berlin: Springer)

Schmalzl, M., Kainulainen, J., Quanz, S. P., et al. 2010, ApJ, 725, 1327

Tapia, S. 1973, in Interstellar Dust and Related Topics, ed. J. M. Greenberg, \& H. C. van de Hulst, IAU Symp., 52, 43

Vassilev, V., Meledin, D., Lapkin, I., et al. 2008, A\&A, 490, 1157

Williams, J. P., de Geus, E. J., \& Blitz, L. 1994, ApJ, 428, 693 